\title{
REDUCED RISK OF PROSTATE CANCER IN MEN WHO ARE CHILDLESS AS COMPARED TO THOSE WHO HAVE FATHERED A CHILD: A POPULATION BASED CASE-CONTROL STUDY
}

Aleksander Giwercman ${ }^{1}$, Lorenzo Richiardi ${ }^{2,3}$, Magnus Kaijser ${ }^{4}$, Anders Ekbom $^{4}$, Olof Akre ${ }^{4}$

1) Fertility Centre, Scanian Andrology Centre, Malmö University Hospital, SE 205 02, Malmö, Sweden

2) Department of Medical Epidemiology, Karolinska Institute, SE-171 77 Stockholm, Sweden

3) Cancer Epidemiology Unit and Center for Oncologic Prevention, CeRMS, University of Turin, I-101

26 Turin, Italy

4) Clinical Epidemiology Unit, Dept. of Medicine, Karolinska Hospital, SE-171 76 Stockholm, Sweden

Corresponding author: Aleksander Giwercman, Fertility Centre, Scanian Andrology Centre, Malmö University Hospital, SE 205 02, Malmö, Sweden. Phone No: +46 40 337904; Fax No: +46 40 337043; email: aleksander.giwercman@kir.mas.lu.se

Key words: prostate cancer; male fertility; children number.

Type: Reasearch article: Epidemiology.

Novelty: The data presented in this paper demonstrate an association between number of children and subsequent risk of prostate cancer. Since male fertility is a marker of testicular function, our findings support a yet unproven notion that a well-functioning testicle during reproductive ages is important in the development of cancer in the human prostate.

Word count: 2,149 words 


\begin{abstract}
(226 words)
Androgens are believed to play a major role in the etiology of prostate cancer, but studies of sexhormone exposure in relation to risk for prostate cancer have been inconclusive. Male fertility may be an indicator of long-term androgen status. To study the role of testicular function in prostate cancer development, we have assessed number of biological children in relation to risk for this malignancy. We performed a population-based case-control study with retrospective ascertainment of cases occurring in Sweden between 1958 and 1998. In total, 48,850 cases of prostate cancer were identified through the nation-wide Cancer Registry. For each case, one control, matched by year of birth, was randomly selected from the Swedish population. Information on offspring was obtained from the Swedish Multi-Generation Registry. We estimated odds ratios (OR) and 95\% confidence intervals (CI) for the association between number of offspring and risk for subsequent prostate cancer. Being childless or having fathered one child only were associated with reduced risks for prostate cancer compared to having fathered two or more children (OR, 0.83; 95\% CI, 0.81-0.86 and OR, 0.93; 95\% CI, 0.90-0.96, respectively). There was no further change in risk associated with fathering of more than two children.

The risk for prostate cancer is reduced among childless men. A dysfunctioning reproductive apparatus fuelling to a lesser extent prostatic growth, could be a plausible mechanism of this association.
\end{abstract}




\section{INTRODUCTION}

Prostate cancer is among the most common cancers worldwide (1). There is a 30 -fold variation in incidence rate between different countries and ethnic groups, but studies on migrants have suggested important environmental causes(1). Despite an astounding number of studies, however, the etiology remains elusive(1).

Etiologic research on prostate cancer has to a great extent been focused on the effect of exposure to sex hormones, mainly androgens. The prostate is one of the main target organs for testosterone, and it is generally believed that androgens are necessary for prostatic carcinogenesis(2). Results from animal and experimental studies have supported this notion $(1 ; 2 ; 3)$ Data on sex-hormonal exposure and risk of prostate cancer in humans are, however, both inconsistent and inconclusive $(1 ; 2)$ possibly because of lack of knowledge of the time period when hormones play their role, and of difficulties in assessing hormonal exposures retrospectively. Unfortunately, there is a lack of suitable direct clinical markers of longstanding androgen activity.

Male infertility is pathogenetically heterogeneous, but associated with testicular failure and impaired androgen secretion(4;5) Thus, the sperm output, and thereby fertility, of a male may be an indicator of his long-term androgen status. We hypothesized that if androgens play a role in prostate cancer etiology, men who have fathered children should be at higher risk compared to childless men. To test this hypothesis, we used Swedish nation-wide registries to assess male fecundity in relation to risk of prostate cancer in a case-control study encompassing more than 48,000 cases and an equal amount of controls.. 


\section{METHODS}

\section{Registry descriptions}

All Swedish residents alive in 1947 onwards have been assigned a 10-digit national registration number, which is a unique personal identifier, referred to in all medical records and official registries. Through the use of the national registration number it is possible to unambiguously link information from several databases together.

Since 1958, all newly diagnosed malignant tumors in Sweden must be reported to the National Cancer Registry, by the physician who makes the diagnosis as well as the pathologist or cytologist who confirms it. All patients are entered in the Cancer Registry by use of their national registration number.

The Register of Population and Population Changes contains the official Swedish census data in a computerized fashion since 1960(6). All residents in Sweden alive at the end of each year are included. Since 1969, the Register also contains information on dates of emigration and/or immigration.

In 2000, Statistics Sweden started a linkage between several data sources from the national registration and created the Swedish Multi-Generation Register(7). The Register contains information on the parents of all individuals in Sweden born in 1932 onwards who survived until 1961. It is thus possible to obtain information on number of children of each index person. For those alive after 1990, the Register is virtually complete with respect to parents as well as offspring. Before 1990, the completeness of registration is dependent on survival: information on parents is lacking for 50 to $60 \%$ of those deceased in the 1960 s, and for 40 to $60 \%$ of those deceased in the $70 \mathrm{~s}$ and $80 \mathrm{~s}(7)$. This incompleteness implicates that the number of children of the 
study subjects may be underestimated in families where subjects died before 1990. It should be noted that no index subject is born before 1932. Adoption or other non-biological relations are flagged in the Register.

\section{Selection of cases and controls}

Through the Cancer Registry, we identified all men (48,910 subjects) born in 1916 onwards who have been diagnosed with prostate cancer (code 177, International Classification of

Diseases, $7^{\text {th }}$ revision) between 1958 and 1998. The reason for excluding those born before 1916 was that the Multi-Generation Registry starts with the birth cohort of 1932, and we intended to cover the subjects' entire reproductive careers. Through the Register of Population and Population Changes, we identified one control for each case, matched by sex, and year and county of birth. Controls had to be alive and without a diagnosis of prostate cancer at the time of diagnosis of the corresponding case. Furthermore, 60 men who, according to the Register of Population and Population Changes, had emigrated were excluded, because of incomplete follow up with respect to fathering and cancer development, leaving 48,850 cases for analysis..

The study has been approved by the ethics committee at Karolinska Institute, Stockholm, Sweden.

\section{Fecundity data}

Using the Swedish Multi-generation Registry we obtained information on date of birth of offspring fathered between 1932 and 1998 by cases and controls. Children of subjects born within five years before the case's date of diagnosis and non-biological children were excluded. Because of the matched design, exclusion of a case or a control led to mutual exclusion of the corresponding case or control. 


\section{Data analysis}

The date of diagnosis for each case was used as time reference for the matched corresponding control. We estimated odds ratios $(\mathrm{OR})$ and $95 \%$ confidence intervals $(\mathrm{CI})$ for the association between number of offspring and risk for subsequent prostate cancer by modeling the data through conditional logistic regression, using the SAS ${ }^{\circledR}$ System software package, version 8. We stratified the data by birth cohort, age at diagnosis, and year of diagnosis among cases to evaluate the potential role of information bias, confounding, or effect modification. Patients diagnosed with prostate cancer at the time of death were included. Results were unchanged when this group was excluded. 


\section{RESULTS}

During the period when subjects were followed up in the Multi-Generation Registry, cases and controls fathered 92,044 and 88,871 children, respectively. Table 1 shows the distribution of the study subjects with respect to year of birth, year of diagnosis, and age at diagnosis. Being childless or having fathered one child only were associated with reduced risks for prostate cancer compared to having fathered two or more children (OR, 0.83; 95\% CI, 0.81-0.86 and OR, 0.93; 95\% CI, 0.90-0.96, respectively) (Table 2). There was no further change in risk associated with fathering of more than two children (data not shown). Stratification by year of birth, year of diagnosis or age at diagnosis did not reveal any substantial heterogeneity (Table 3). 


\section{DISCUSSION}

This study is by far the largest to assess fecundity in relation to risk of prostate cancer risk. We found that childless men have a slightly reduced risk compared with those who have fathered one or more children. Being childless may be a result of impaired sperm production, and our results thus may indicate that testicular dysfunction in reproductive ages is protective against cancer development in the prostate.

Through the use of registries, we were able to perform a large nationwide case-control study with identical data collection from cases and controls. Our ideal aim would have been to assess male fertility status which is, however, difficult if not impossible to measure with high validity in a population-based study of risk for prostate cancer. Fecundity is an indirect measure of male fertility, encompassing variables such as fertility of the female partner and social factors influencing the desire or ability to have children. The group of childless men, thus, also includes individuals who in fact did have a normal reproductive capacity. Such misclassification, however, introduces a non-differential bias that most probably should result in an underestimation of the association with fertility, rather than produce a false positive result. Therefore, we conclude that our findings demonstrate an association between degree fertility and risk of prostate cancer, an interpretation supported by the slightly decreased risk among men who have fathered one child compared to those having two or more children. Male fertility may, on the population level be a determinant of family size in families with one child, but should be a less important determinant for larger family sizes, which are mainly dependent on social and cultural factors(8).

We do have a problem with misclassification of number of children since the multigeneration register has a survival-dependent drop out that varies over time with underreporting of the offspring that died before 1991 (7). However, we found homogeneity over different time- 
dependent strata, including recent birth cohorts and strata where cases were diagnosed after 1990 implicating that such underreporting has influenced the results marginally.

Another shortcoming of our study is the lack of control of potential confounders as dietary factors and socioeconomic status. We have, however, no strong suspicion of confounding by diet, since the association between dietary factors and fecundity supposedly is weak, and no specific dietary factor is known to cause a substantial proportion of prostate cancer cases. Socioeconomic status is not controlled for in the present analysis, but there is, in the Swedish population, no established association between socioeconomic status and prostate cancer, which would be required for a confounding effect(9). Some studies(10) but not all(11) have indicated a decreased risk for prostate cancer among unmarried men, compatible with present data. It is conceivable that husbands and fathers are more prone to screening behavior, but it should be kept in mind that the vast majority of cancer cases included in this study was diagnosed before the screening for prostate cancer became available.

Although fertility and fecundity has received little attention in etiologic research on prostate cancer, it has been assessed by a number of previous studies(12). Few or none of them had reasonable statistical power to detect relative risks of the magnitude reported in the present study. However, in a recent meta-analysis(12) of measures of sexual activity and prostate cancer risk, the pooled estimate from 24 previous studies indicated a weak association (relative risk, 1.07; 95\% CI, 0.97-1.18 for four children compared with none; $\mathrm{p}$ for trend with increasing number of children, 0.0003) with number of children that was attributable only to data from hospital-based case-control studies.

Our results may throw a new light on previously reported association between vasectomy and the risk for prostate cancer(13). A convincing biological model for such association has never been given(14) Vasectomy is, however, virtually never done among childless men and it could 
thus be speculated that the association with vasectomy reflects a biological link between male fertility and risk for prostate cancer.

Androgens are essential for normal growth and function of the prostate and may play an important role in prostatic carcinogenesis(2). The vast majority of tumors initially responds to anti-androgenic therapy with temporary remission, but relapses to a hormone-refractory state at later stages(15). Laboratory data have given further support to the role of androgens, but data from numerous serological studies, both of prospective and retrospective design, are inconclusive and the role of androgens in the etiology of prostate cancer remains unclear( $1 ; 2)$. Several unsolved methodological problems may explain the lack of evidence in humans. First, studies on humans should preferably use prospectively collected specimens to preclude reverse causality, and only a few repositories are of sufficient size to enable studies with cancer as outcome. Second, the development of prostate cancer may be a result of a long-term androgen action and few specimen banks contain repeated measurements to enable studies of different age periods. Third, circulating androgens may not correspond well to the local androgenic activity in the prostate, which may, for example, be regulated by the local activity of $5 \alpha$-reductase converting testosterone to the more potent androgen dihydrotestosterone, and by receptor sensitivity to sex hormones(2). Thus, lack of evidence from studies of hormones in serum is not evidence against the role of hormones in prostate cancer development.

Testicular failure is the main cause of impaired male fertility(4). Decreased sperm production and fertility problems are frequently accompanied by androgen deficiency(16). Furthermore, impairment of sperm production has been etiologically linked to Leydig cell dysfunction as a part of Testicular Dysgenesis Syndrome(17) Our finding of an association between fecundity and PC 
risk is thus consistent with the hypothesis that functioning gonads fuel prostatic carcinogenesis by androgen secretion.

The association with number of children may also reflect genetically determined difference in androgen sensitivity between cases and controls. Androgen sensitivity is regulated by the length of the glutamine encoding CAG repeat of exon 1 of the androgen receptor gene (AR)(18). Extremely long repeats of more than 40 CAG sequences leads to Kennedy's disease, which presents with neuromuscular symptoms, hypogonadism and impaired sperm production(19). However, even within the normal range, that is, between 15 and 30 repeats, there seems to be a negative correlation between the repeat number and androgen sensitivity, sperm production and fertility $(18 ; 20 ; 21)$. Average number of CAG repeats is inversely correlated with the incidence of PC in different ethnic groups: African-American men, who have a high incidence of PC, have shorter mean CAG sequences (mean: 18-19) than Caucasians (mean: 22) and Asians (mean: 23) who have lower and the lowest PC incidences, respectively(22). Some analytical studies have also shown an increased risk of PC among males with low number of CAG repeats $(23 ; 24)$ although evidence is equivocal(22).

In conclusion, the present study reports evidence of an association between fecundity and risk for PC. The finding supports the common but yet unproven notion that a well-functioning testicle is important in the development of cancer in the human prostate. 


\section{ACKNOWLEDGEMENT}

Supported by the Swedish Cancer Society (Grant No: 00 0367), Gunnar Nielsens Cancer Fund, Malmö University Hospital Cancer Fund, and Swedish governmental fund for clinical research.

The work of Lorenzo Richiardi was in part supported by the Special project "Oncology" Compagnia San paolo / FIRMS 


\section{Reference List}

1. Signorello LB, Adami H.-O., Prostate cancer, In: H.-O. Adami, D. J. Hunter, and D. Trichopoulos (eds.), Textbook of cancer epidemiology, pp. 400-419, Oxford University Press, New York, (2002).

2. Kaaks R, Lukanova, A,Sommersberg, B. Plasma androgens, IGF-1, body size, and prostate cancer risk: a synthetic review. Prostate Cancer Prostatic Dis 2000;3:157-172.

3. Eng MH, Charles, LG,Ross, BD,Chrisp, CE,Pienta, KJ,Greenberg, NM,Hsu, CX,Sanda, MG. Early castration reduces prostatic carcinogenesis in transgenic mice. Urology $1999 ; 54: 1112-1119$.

4. Skakkebæk NE, Giwercman, A,De Kretser, D. Pathogenesis and management of male infertility. Lancet 1994;343:1473-1479.

5. Pavlovich CP, King, P,Goldstein, M,Schlegel, PN. Evidence of a treatable endocrinopathy in infertile men. J Urol 2001;165:837-841.

6. Ett nytt system för Registret över totalbefolkningen: fler möjligheter och bättre kvalitet. $=$ A new total population register system: more possibilities and better quality., Statistics Sweden, Stockholm, (2002).

7. Statistiska centralbyrån. Flergenerationsregisteret $=$ The multigeneration register, Statistics Sweden, Stockholm, (2001).

8. ESHRE Capri Workshop Group. Social determinants of human reproduction. Hum Reprod $2001 ; 16: 1518-1526$. 
9. Andersson SO, Baron, J,Bergstrom, R,Lindgren, C,Wolk, A,Adami, HO. Lifestyle factors and prostate cancer risk: a case-control study in Sweden. Cancer Epidemiol Biomarkers Prev 1996;5:509-513.

10. Harvei S, Kravdal, O. The importance of marital and socioeconomic status in incidence and survival of prostate cancer. An analysis of complete Norwegian birth cohorts. Prev Med 1997;26:623-632.

11. Lund Nilsen TI, Johnsen, R,Vatten, LJ. Socio-economic and lifestyle factors associated with the risk of prostate cancer. Br J Cancer 2000;82:1358-1363.

12. Dennis LK, Dawson, DV. Meta-analysis of measures of sexual activity and prostate cancer. Epidemiology 2002;13:72-79.

13. Rosenberg L, Palmer, JR,Zauber, AG,Warshauer, ME,Stolley, PD,Shapiro, S. Vasectomy and the risk of prostate cancer. Am J Epidemiol 1990;132:1051-1055.

14. Bernal-Delgado E, Latour-Perez, J,Pradas-Arnal, F,Gomez-Lopez, LI. The association between vasectomy and prostate cancer: a systematic review of the literature. Fertil Steril 1998;70:191-200.

15. Goodin S, Rao, KV,DiPaola, RS. State-of-the-art treatment of metastatic hormonerefractory prostate cancer. Oncologist 2002;7:360-370.

16. Andersson AM, Jorgensen, N,Frydelund-Larsen, L,Rajpert-De Meyts, E,Skakkebaek, NE. Impaired Leydig cell function in infertile men: a study of 357 idiopathic infertile men and 318 proven fertile controls. J Clin Endocrinol Metab 2004;89:3161-3167. 
17. Skakkebæk NE, Rajpert-De Meyts, E,Main, KM. Testicular dysgenesis syndrome: an increasingly common developmental disorder with environmental aspects. Hum Reprod $2001 ; 16: 972-978$.

18. Tut TG,Ghadessy, FJ,Trifiro, MA,Pinsky, L, Young, EL. Long polyglutamine tracts in the androgen receptor are associated with reduced trans-activation, impaired sperm production and male infertility. JCEM 1997;82:3777-3782.

19. Van Golde R, Van Houwelingen, K,Kiemeney, L,Kremer, J,Tuerlings, J,Schalken, J,Meuleman, E. Is increased CAG repeat length in the androgen receptor gene a risk factor for male subfertility? J Urol 2002;167:621-623.

20. von Eckardstein S, Syska, A,Gromoll, J,Kamischke, A,Simoni, M,Nieschlag, E. Inverse correlation between sperm concentration and number of androgen receptor CAG repeats in normal men. JCEM 2001;86:2585-2590.

21. Dowsing AT, Yong, EL,Clark, M,McLachlan, RI,de Kretser, DM,Trounson, AO. Linkage between male infertility and trinucleotide repeat expansion in the androgen receptor gene. The Lancet 1999;354:640-643.

22. Nelson KA, Witte, JS. Androgen receptor CAG repeats and prostate cancer. Am J Epidemiol 2002;155:883-890.

23. Mononen N, Ikonen, T,Autio, V,Rokman, A,Matikainen, MP,Tammela, TL,Kallioniemi, OP,Koivisto, PA, Schleutker, J. Androgen receptor CAG polymorphism and prostate cancer risk. Hum Genet 2002;111:166-171. 
24. Balic I,Graham, ST, Troyer, DA,Higgins, BA,Pollock, BH,Johnson-Pais, TL,Thompson, IM,Leach, RJ. Androgen receptor length polymorphism associated with prostate cancer risk in Hispanic men. J Urol 2002;168:2245-2248. 
Table 1. Distribution of 48,850 prostate cancer cases* in Sweden, diagnosed between 1958 and 1998.

number of cases $\quad \%$

Year of birth

$\begin{array}{lrr}-1919 & 15681 & 32.1 \\ 1920-1924 & 16249 & 33.3 \\ 1925-1929 & 9005 & 18.4 \\ 1930-1934 & 4727 & 9.7 \\ 1935+ & 3188 & 6.5\end{array}$

Year of diagnosis

$\begin{array}{lrr}-1974 & 555 & 1.1 \\ 1975-1979 & 1421 & 2.9 \\ 1980-1984 & 3621 & 7.4 \\ 1985-1989 & 8456 & 17.3 \\ 1990-1994 & 15690 & 32.1 \\ 1995+ & 19107 & 39.1\end{array}$

Age at diagnosis

$\begin{array}{lrr}-49 & 408 & 0.8 \\ 50-59 & 5130 & 10.5 \\ 60-69 & 20032 & 41.0 \\ 70+ & 23280 & 47.7\end{array}$

\footnotetext{
*Numbers of controls were identical.
} 
Table 2. Adjusted odds ratios and 95 percent confidence intervals for the association between number of children and risk of prostate cancer.

\begin{tabular}{lccc}
\hline Number of children & Cases & Controls & Odds ratio* $(95 \%$ CI) \\
& & & \\
0 & $9476(19.4)$ & $10790(22.1)$ & $0.83(0.81-0.86)$ \\
1 & $9380(19.2)$ & $9564(19.6)$ & $0.93(0.90-0.96)$ \\
$2+$ & $29994(61.4)$ & $28496(58.4)$ & 1.00 (reference) \\
$*$ *Rs adjusted for year of birth and age at diagnosis. &
\end{tabular}


Table 3. Odds ratios* and 95\% confidence intervals (CIs) for the association between number of children and risk of prostate cancer, stratified by year of birth, year of diagnosis, and age at diagnosis.

\section{Odds Ratio* $(95 \% \mathrm{CI})$}

Number of children

\begin{tabular}{lll}
\hline 0 & 1 & $2+$
\end{tabular}

Year of birth

$-1919$

$1920-1924$

1925-1929

$1930-1934$

$1935+$

Year of diagnosis

$-1974$

1975-1979

1980-1984

1985-1989

1990-1994

$1995+$

Age at diagnosis
$1.05(0.79-1.40)$

$0.96(0.80-1.15)$

$0.88(0.78-0.98)$

$0.86(0.80-0.93)$

$0.83(0.78-0.87)$

$0.81(0.76-0.85)$

$-49$

50-59

60-69

$70+$
$1.08(0.76-1.54)$

$0.90(0.81-1.00)$

$0.80(0.76-0.84)$

$0.84(0.81-0.88)$
$0.98(0.92-1.03)$

$0.90(0.85-0.95)$

$0.96(0.89-1.04)$

$0.84(0.75-0.93)$

$0.94(0.82-1.08)$
1.00 (reference)

1.00 (reference)

1.00 (reference)

1.00 (reference)

1.00 (reference)
$1.04(0.77-1.39)$

$1.07(0.89-1.30)$

$1.02(0.91-1.15)$

$0.93(0.86-1.00)$

$0.91(0.86-0.96)$

$0.92(0.88-0.97)$

1.00 (reference)

1.00 (reference)

1.00 (reference)

1.00 (reference) 